\title{
Convergence Theorems for Renormalized Feynman Integrals with Zero-Mass Propagators
}

\author{
J. H. Lowenstein * \\ Physics Department, New York University, New York, N.Y. 10003, USA ${ }^{\star \star}$, and \\ Max-Planck-Institut für Physik und Astrophysik, D-8000 München, Federal Republic of Germany
}

\begin{abstract}
A general momentum-space subtraction procedure is proposed for the removal of both ultraviolet and infrared divergences of Feynman integrals. Convergence theorems are proved which allow one to define time-ordered Green functions, as tempered distributions, for a wide class of theories with zero-mass propagators.
\end{abstract}

\section{Introduction}

Until recently, the applicability of Zimmermann's momentum-space subtraction procedure $[1,2]$ for Feynman integrals was limited to cases with no vanishing masses. To be sure, zero-mass theories could be discussed, but only as limits of corresponding massive theories, and even then only the limits for non-exceptional Euclidean momenta could be taken legitimately. The aim of the present work is to extend the convergence theorems of Zimmermann to a very general class of momentum-space Feynman integrals with arbitrary non-negative mass parameters.

The subtraction scheme adopted below evolved from one used by the author and Zimmermann [3] to formulate the massless $A^{4}$ model (see also [4]). The essential idea is the following: all subtractions are made at vanishing momentum, but some of them, including the ones otherwise expected to give rise to infrared divergences (mass singularities) by naive power counting, are made at a non-zero value of the mass. The absolute convergence of the subtracted Feynman integrals in the massless $A^{4}$ model has been proved [5] for non-exceptional (in the Euclidean sense) external momenta and non-zero $\varepsilon$ in the denominator factors. Similar treatments have been given for the Goldstone model and Abelian Higgs model $[3,6]$. Unfortunately the methods of these references are not sufficient to treat models with renormalization parts with three external boson lines (e.g. massless scalar QED, non-Abelian Yang-Mills models). If one gives the three-

* Research supported in part by National Science Foundation Grant MPS-74-21778.

$\star \star$ Permanent address. 
vertices extra subtractions at zero mass, as one must to satisfy the hypothesis of the general power-counting theorem [7], one runs into the problem of overlaps. As we shall see, this problem can be handled in a manner very similar to that used by Zimmermann [1] to verify the ultraviolet power-counting criterion.

The main convergence theorems presented below (Section III) provide relatively simple criteria for the absolute convergence of the following two integrals involving $R_{\Gamma \varepsilon}(p, k)$ the Feynman integrand for a diagram $\Gamma$, subtracted according to our new prescription (specified in Section II):

$$
\begin{aligned}
& \int d^{4 m} k d^{4 n} p f(p) R_{\Gamma \varepsilon}(p, k) \\
& \quad \Gamma \quad \text { connected } \\
& \varepsilon>0 \\
& \quad f \in \mathscr{S}\left(\mathbb{R}^{4 n}\right) \\
& \text { and } \\
& \quad \int d^{4 m} k R_{\Gamma \varepsilon}(p, k) \\
& \quad \Gamma \text { one-particle irreducible } \\
& \quad \varepsilon>0 \\
& \quad\left\{p_{i}\right\} \text { non-exceptional (Euclidean sense) }
\end{aligned}
$$

The usefulness of these theorems lies in their applicability to the definition, as tempered distributions, of the time-ordered Green functions of a wide class of quantum fields and their normal products. The absolute convergence of each Feynman integral contributing to such a Green function is shown in Section IV below; the final distributional limit and the proof of Lorentz invariance are presented elsewhere [8]. It can be shown [9] that the normal products defined by this procedure have all of the convenient properties needed for the systematic derivation of Ward identities and other structural relations of perturbative Lagrangian field theory. Already these normal products have proven extremely useful in the formulation, with the Bogoliubov-Parasiuk-Hepp-Zimmermann framework, of the massless Yang-Mills model $[10,11]$ and the Georgi-Glashow model [12].

\section{Preliminary Considerations}

The momentum-space integrand (without subtractions) for a connected Feynman diagram $\Gamma$ is assumed to be of the form

$$
I_{\Gamma \varepsilon}(p, k, s)=\prod_{V \in \Gamma} P_{V}\left(\left\{l_{V i}\right\}, s\right) \prod_{L \in \Gamma} \Delta_{L}\left(l_{L}, s\right)
$$


where

$$
\begin{aligned}
& p=\left\{p_{1}, p_{2}, \ldots, p_{n}\right\}=\text { basis for external momenta of } \Gamma \\
& k=\left\{k_{1}, k_{2}, \ldots, k_{n}\right\}=\text { basis for internal momenta of } \Gamma \\
& 0 \leqq s \leqq 1 \\
& \prod_{V \in \Gamma}=\text { product over vertices } V \text { of } \Gamma \\
& \prod_{L \in \Gamma}=\text { product over lines } L \text { of } \Gamma \\
& \left\{l_{V i}\right\}=\text { set of momenta flowing into vertex } V \\
& l_{L}=\text { momentum flowing through line } L \\
& \Delta_{L}\left(l_{L}, s\right)=P_{L}\left(l_{L}, s\right) / \prod_{i}\left[F_{L i}\left(l_{L}, s\right)\right]^{v_{i}} \\
& F_{L i}\left(l_{L}, s\right)=l_{L}^{2}-(s-1)^{2} M_{L i}^{2}-s^{2} \mu_{L i}^{2}-m_{L i}^{2}+i \varepsilon\left(l_{L}^{2}+(s-1)^{2} M_{L i}^{2}+s^{2} \mu_{L i}^{2}+m_{L i}^{2}\right) \\
& m_{L i}^{2}+M_{L i}^{2}>0 \\
& P_{L}=\text { polynomial in } l_{L} \text { and } s \\
& v_{i}=\text { positive integer } .
\end{aligned}
$$

Of particular importance in any subtraction procedure for Feynman integrals are the one-particle irreducible (1PI) sub-diagrams of a diagram $\Gamma$. With each 1PI $\gamma$ we associate ultraviolet (UV) and infrared (IR) superficial divergences, suitably generalized to incorporate the parameter $s$, given by

where

$$
\begin{aligned}
& d(\gamma)=4 m(\gamma)+\sum_{V \in \gamma} \bar{D}_{V}+\sum_{L \in \gamma} \bar{D}_{L} \\
& r(\gamma)=4 m(\gamma)+\sum_{V \in \gamma} \underline{D}_{V}+\sum_{L \in \gamma} \underline{D}_{L}
\end{aligned}
$$

$$
\begin{array}{ll}
\bar{D}_{L}=\overline{\operatorname{deg}}_{p s} \Delta_{L}, & \underline{D}_{L}=\underline{\operatorname{deg}}_{p(s-1)} \Delta_{L} \\
\bar{D}_{V}=\overline{\operatorname{deg}}_{p s} P_{V}, & \underline{D}_{V}=\underline{\operatorname{deg}}_{p(s-1)} P_{V}
\end{array}
$$

and $m(\gamma)$ is the number of independent $l_{L}, L \in \gamma$, for fixed external momenta of $\gamma$. The upper degree $\overline{\operatorname{deg}}_{p s}$ gives the asymptotic power for $p$ and $s$ tending to infinity; the lower degree $\operatorname{deg}_{p(s-1)}$ gives the asymptotic power for $p$ and $s-1$ tending to zero [see $(2.15-16$ below].

Using the identity

$$
m(\gamma)=(\text { no. of } L \in \gamma)-(\text { no. of } V \in \gamma)+1 \text {, }
$$

equations (2.2) may be rewritten as

$$
\begin{aligned}
& d(\gamma)=4+\sum_{V \in \gamma}\left(\bar{D}_{V}-4\right)+\sum_{L \in \gamma}\left(\bar{D}_{L}+4\right) \\
& r(\gamma)=4+\sum_{V \in \gamma}\left(\underline{D}_{V}-4\right)+\sum_{L \in \gamma}\left(\underline{D}_{L}+4\right) .
\end{aligned}
$$


A reduced subdiagram $\Lambda / \lambda_{1} \ldots \lambda_{n}$ is obtained from $\Lambda$ by contracting mutually disjoint, non-trivial 1 PI subgraphs $\lambda_{i}$ to points [reduced vertices $V\left(\lambda_{i}\right)$ ]. Assigning the unit polynomial to each $V\left(\lambda_{i}\right)$, we see that for 1 PI $\gamma$ and disjoint $\lambda_{i} \subset \gamma$,

$$
\begin{aligned}
& d(\gamma)=d\left(\gamma / \lambda_{1} \ldots \lambda_{n}\right)+\sum_{i=1}^{n} d\left(\lambda_{i}\right) \\
& r(\gamma)=r\left(\gamma / \lambda_{1} \ldots \lambda_{n}\right)+\sum_{i=1}^{n} r\left(\lambda_{i}\right) .
\end{aligned}
$$

Although the UV and IR degrees are applied only to 1PI diagrams in the subtraction procedure, it is obvious that the concepts can be generalized to connected diagrams, with formulae (2.2-4) remaining valid. This generalization will be employed in stating sufficient conditions for infrared convergence in Theorems 3.1 and 3.2.

More generally, one assigns to each $1 \mathrm{PI} \gamma \subseteq \Gamma$ ultraviolet and infrared subtraction degrees, $\delta(\gamma)$ and $\varrho(\gamma)$, defined respectively by

$$
\begin{aligned}
& \delta(\gamma)=d(\gamma)+b(\gamma) \\
& \varrho(\gamma)=r(\gamma)-c(\gamma)
\end{aligned}
$$

where $b(\gamma)$ and $c(\gamma)$ are non-negative integers constrained by the inequalities

$$
\begin{aligned}
& \delta(\gamma) \geqq d\left(\gamma / \lambda_{1} \ldots \lambda_{n}\right)+\sum_{i=1}^{n} \delta\left(\lambda_{i}\right) \\
& \varrho(\gamma) \leqq r\left(\gamma / \lambda_{1} \ldots \lambda_{n}\right)+\sum_{i=1}^{n} \varrho\left(\lambda_{i}\right) \\
& \varrho(\gamma) \leqq \delta(\gamma)+1
\end{aligned}
$$

for arbitrary reduced 1PI subdiagram $\gamma /\left\{\lambda_{i}\right\}$ of $\Gamma$.

The subtracted integrand for $\Gamma$ is defined by a modified version of Zimmermann's "forest formula" [13],

$$
R_{\Gamma \varepsilon}(p, k, s)=S_{\Gamma} \sum_{U \in \mathscr{F}_{\Gamma}} \prod_{\gamma \in U}\left(-\tau_{\gamma} S_{\gamma}\right) I_{\Gamma \varepsilon}(U),
$$

where $\mathscr{F}_{\Gamma}$ is the set of $\Gamma$-forests (families of non-trivial, non-overlapping 1PI subgraphs of $\Gamma$ );

$I_{\Gamma \varepsilon}(U)$ is the unsubtracted integrand (2.1) in which $l_{V i}$ and $l_{L}$ are written

$$
\begin{gathered}
l_{V i}^{\tau}=q_{V i}^{\tau}\left(p^{\tau}\right)+k_{V i}^{\tau}(k) \\
l_{L}^{\tau}=q_{L}^{\tau}\left(p^{\tau}\right)+k_{L}^{\tau}(k)
\end{gathered}
$$

where $\tau$ is the smallest element of $U \cup\{\Gamma\}$ containing $V$ (resp. $L$ ). Here $q_{V i}^{\tau}$ (resp. $q_{L}^{\tau}$ ) is a linear function of the external momenta $p_{j}^{\tau}$ of $\tau$, considered as independent variables, and $k_{V i}^{\tau}$ (resp. $k_{L}^{\tau}$ ) is a linear function of the internal momenta of $\Gamma$. Similarly, the variable $s$ appearing in $P_{V}$ or $\Delta_{L}$ is replaced by $s^{\tau}$;

$S_{\gamma}$ is a substitution operator, shifting from the variables of $\lambda \in U$ to thiose of $\gamma \in U, \gamma \supset \lambda$;

$\tau_{\gamma}$ is the subtraction operator defined by

$$
\left(1-\tau_{\gamma}\right)=\left(1-t_{p^{\gamma}\left(s^{\gamma}-1\right)}^{e(\gamma)-1}\right)\left(1-t_{p^{\gamma /} s^{\gamma}}^{\delta(\gamma)}\right.
$$


where, in general,

$$
t_{x_{1} \ldots x_{n}}^{d}=\left\{\begin{array}{l}
\text { Taylor series about } x_{i}=0 \text { to order } \\
d \text { if } d \geqq 0 \\
0 \text {. if } d<0
\end{array}\right.
$$

The above description of unsubtracted and subtracted Feynman integrands assumes some familiarity on the part of the reader with Zimmermann's formalism. A more complete explanation of the nomenclature may be found in Ref. [1].

Having specified the subtraction rules for constructing renormalized integrands, we now want to lay the groundwork for the absolute convergence theorems of Section III. We first observe that the absolute convergence of the integral (1.1) is implied by that of

$$
\int d p d k R_{\hat{\Gamma} \varepsilon}(p, k, s)
$$

where $R_{\hat{\Gamma} \varepsilon}$ is the subtracted integrand for the augmented diagram $\hat{\Gamma}$ obtained from $\Gamma$ by drawing special lines ( $q$-lines) which carry the external momenta $q_{j}(p)$ and which meet in a new internal vertex $V_{0}$. In computing $R_{\hat{\Gamma} \varepsilon}$, each $q$-line of $\hat{\Gamma}$ is assigned a propagator

$$
\left(q_{i}(p)^{2}-\mu^{2}+i \varepsilon\left(q_{i}^{2}+\mu^{2}\right)\right)^{-v}, \quad i=1,2, \ldots, n+1
$$

with $\mu^{2}>0$ and $v$ chosen sufficiently large that both $\varrho(\gamma)$ and $\delta(\gamma)$ may be taken to be negative [and consistent with (2.6)] for every 1PI subgraph $\gamma$ of $\hat{\Gamma}$ which contains $V_{0}$. The foregoing construction of $\hat{\Gamma}$ is of course not applicable to a case where $\Gamma$ has only one external line (tadpole) or no external lines (vacuum bubble). In the former case we define $\hat{\Gamma}$ to be $\Gamma$ with its external line amputated; in the latter case we simple set $\hat{\Gamma} \equiv \Gamma$. Thus in all cases $\hat{\Gamma}$ is a connected diagram with only internal vertices.

We now restate the main theorem of Ref. [7] with a somewhat stronger (but simpler) hypothesis:

Theorem 2.[7]. Let $R(p, k)$ be a function of four-vector variables $p=p_{1} \ldots p_{n}$ and $k=k_{1} \ldots k_{m}$ of the form

$$
R(p, k)=P(p, k) / \prod_{i}\left(l_{i}^{2}-m_{i}^{2}+i \varepsilon\left(\boldsymbol{l}_{i}^{2}+m_{i}^{2}\right)\right)
$$

where $P(p, k)$ is a polynomial in the components of $p$ and $k$ and each $l_{i}$ is an element of $\mathscr{L}$, the space of linear forms

$$
\sum_{i=1}^{n} \alpha_{i} p_{i}+\sum_{j=1}^{m} \beta_{j} k_{j}
$$

The integral

$$
\int d^{4 m} k R(p, k)
$$


converges absolutely if for every choice of $u_{i} \in \mathscr{L}, i=1,2, \ldots, a$ and $v_{j} \in \mathscr{L}$, $j=1,2, \ldots m-a$ such that the Jacobian $\partial(u, v) / \partial(k)$ is non-vanishing, the following $U V$ and IR convergence conditions are satisfied:

$$
\begin{aligned}
& \overline{\operatorname{deg}}_{u \mid v} R(p, k(u, v, p))+4 a<0 \\
& \underline{\operatorname{deg}}_{u \mid v} R(p, k(u, v, p))+4 a>0 .
\end{aligned}
$$

The upper and lower degrees are defined as in [7]. For a function $F(x, y)$ of variables $x_{1} \ldots x_{b}, y_{1} \ldots y_{c}$ we have

$$
\begin{aligned}
& \overline{\operatorname{deg}}_{x \mid y} F=h \\
& \operatorname{deg}_{x \mid y} F=k
\end{aligned}
$$

if, for almost all $x_{1} \ldots x_{b}, y_{1} \ldots y_{c}$,

$$
\begin{aligned}
& \lim _{\lambda \rightarrow \infty} \lambda^{-h} F(\lambda x, y) \neq 0, \infty \\
& \lim _{\lambda \rightarrow 0} \lambda^{-k} F(\lambda x, y) \neq 0, \infty .
\end{aligned}
$$

Some of the important properties of these degree functions are

$$
\begin{aligned}
& \overline{\operatorname{deg}}_{x \mid y} \prod_{j=1}^{r} F_{j}=\sum_{j=1}^{r} \overline{\operatorname{deg}}_{x \mid y} F_{j} \\
& \underline{\operatorname{deg}}_{x \mid y} \prod_{j=1}^{r} F_{j}=\sum_{j=1}^{r} \underline{\operatorname{deg}_{x \mid y} F_{j}} \\
& \overline{\operatorname{deg}}_{x \mid y} \sum_{j=1}^{r} F_{j} \leqq \max _{j}\left\{\overline{\operatorname{deg}}_{x \mid y} F_{j}\right\} \\
& \underline{\operatorname{deg}}_{x \mid y} \sum_{j=1}^{r} F_{j} \geqq \min _{j}\left\{\operatorname{deg}_{x \mid y} F_{j}\right\},
\end{aligned}
$$

with equality in (2.19-20) if $F_{j}$ are linearly independent monomials in $x$ and $y$.

\section{Convergence Theorems}

We are now ready to state our first main theorem.

Theorem 3.1. Let $\Gamma$ be a connected Feynman diagram, whose subtracted integrand is given by (2.7). Suppose that for every set $\{\gamma\}$ of mutually disjoint, non-trivial $1 P I$ subgraphs of $\hat{\Gamma}$ with $\hat{\Gamma} \notin\{\gamma\}$ the inequality

$$
r(\hat{\Gamma} /\{\gamma\})+\sum_{\gamma} \max \{0, \varrho(\gamma)\}>0
$$

is satisfied. Then the integral

$$
\int d p d k f(p) R_{\Gamma \varepsilon}(p, k, s), \quad 0 \leqq s \leqq 1,
$$

converges absolutely for arbitrary $\varepsilon>0$ and $f \in \mathscr{S}\left(\mathbb{R}^{4 n}\right)$. 
Crucial to our proof of this theorem is the concept of complete forest [1]. Suppose that $S$ is a linear subspace of $\mathscr{L}(\Gamma)$, the space of linear forms in $p$ and $k$, and $\bar{\gamma}$ is a reduced subdiagram of $\Gamma$. We shall say that $\bar{\gamma}$ lies along $S$ (in symbols $\bar{\gamma} \| S$ ) if, for all (internal) lines $L \in \bar{\gamma}, k_{L}^{\gamma} \in S$ [see (2.8)]. If $\bar{\gamma}$ has no line $L$ with $k_{L}^{\gamma} \in S$, we shall say that $\bar{\gamma}$ is oblique to $S$ (in symbols $\bar{\gamma} \nmid S$ ). A $\Gamma$-forest $C$ is complete with respect to $S$ if

$$
\Gamma 1 \mathrm{PI} \Rightarrow \Gamma \in C
$$

and, for all $\gamma \in C \cup\{\Gamma\}$,

$$
\bar{\gamma}(C) \| S \text { or } \bar{\gamma}(C) \times S,
$$

where $\bar{\gamma}(C)$ is $\gamma$ reduced by all elements of $C$ properly contained in it. The usefulness of complete forests derives from the following result of Zimmermann [1]:

Lemma 3.1. [1]. Let $\Gamma$ be a connected diagram, $S$ a subspace of $\mathscr{L}(\Gamma)$; then

$$
\begin{aligned}
R_{\Gamma \varepsilon} & =\sum_{C \in \mathscr{C}_{S}(\Gamma)} R_{\Gamma \varepsilon}(C) \\
R_{\Gamma \varepsilon}(C) & =\left(1-\tau_{\Gamma}\right) Y_{\Gamma}(C)
\end{aligned}
$$

where

$$
\mathscr{C}_{S}(\Gamma)=\left\{U \in \mathscr{F}_{\Gamma}: U \text { complete w.r.t. } S\right\}
$$

and $Y_{\gamma}(C), \gamma \subseteq \Gamma$, is defined recursively by

$$
\begin{aligned}
& Y_{\gamma}(C)=I_{\bar{\gamma}(C)} S_{\gamma} \prod_{\alpha} f_{\gamma_{\alpha}} Y_{\gamma_{\alpha}}(C) \\
& \bar{\gamma}(C)=\gamma / \gamma_{1} \ldots \gamma_{n} \\
& \left\{\gamma_{1}, \ldots, \gamma_{n}\right\}=\text { set of maximal elements of } C \text { contained in } \gamma \\
& f_{\gamma_{\alpha}}=\left\{\begin{array}{l}
1-\tau_{\gamma_{\alpha}} \text { if } \bar{\gamma}_{\alpha}(C) \times S, \bar{\gamma}(C) \| S \\
-\tau_{\gamma_{\alpha}} \text { otherwise. }
\end{array}\right.
\end{aligned}
$$

The subtraction operator $\tau_{\Gamma}$ is, by convention, non-zero only if $\Gamma$ is $1 P I$ and $\delta(\Gamma) \geqq 0$.

We now present three power-counting lemmas which will be used in proving Theorems 3.1 and 3.2. The first of these lists those properties of the subtraction operator $\tau_{\gamma}$ which are relevant to infrared power counting.

Lemma 3.2. Let $u_{1}, \ldots, u_{a}, v_{1}, \ldots, v_{b}, a>0$, be an arbitrary basis of $\mathscr{L}(\Gamma)$, the space of linear forms in the internal and external momenta of a connected graph $\Gamma$. Furthermore, let $C$ be a $\hat{\Gamma}$-forest which is complete with respect to $S$, the subspace of $\mathscr{L}(\Gamma)$ spanned by $u_{1}, \ldots u_{a}$. 
For $\tau_{\gamma} Y_{\gamma}(C) \neq 0$, we have

$\underline{\operatorname{deg}}_{u p^{\gamma}\left(s^{\gamma}-1\right) \mid v} \tau_{\gamma} Y_{\gamma}(C)$

$\geqq \min \left\{\underline{\operatorname{deg}}_{u p^{\gamma}\left(s^{\gamma}-1\right) \mid v} Y_{\gamma}(C), \operatorname{deg}_{u \mid p^{\gamma}\left(s^{\gamma}-1\right) v} Y_{\gamma}(C)+\max \{0, \varrho(\gamma)\}\right\}$,

$\underline{\operatorname{deg}}_{u \mid p^{\gamma}\left(s^{\gamma}-1\right) v} \tau_{\gamma} Y_{\gamma}(C)$

$$
\begin{aligned}
\geqq & \left\{\begin{array}{l}
\operatorname{deg}_{u \mid p^{\gamma}\left(s^{\gamma}-1\right) v} Y_{\gamma}(C) \quad \text { if } \quad \bar{\gamma}(C) \times S \\
\min \left\{\operatorname{deg}_{u p^{\gamma}\left(s^{\gamma}-1\right) \mid v} Y_{\gamma}(C)-\varrho(\gamma)+1, \underline{\operatorname{deg}}_{u \mid p^{\gamma}\left(s^{\gamma}-1\right) v} Y_{\gamma}(C)\right\}
\end{array}\right. \\
& \text { if } \bar{\gamma}(C) \| S,
\end{aligned}
$$
$\underline{\operatorname{deg}}_{u p^{\gamma}\left(s^{\gamma}-1\right) \mid v}\left(1-\tau_{\gamma}\right) Y_{\gamma}(C) \geqq \operatorname{deg}_{u \mid p^{\gamma}\left(s^{\gamma}-1\right) v} Y_{\gamma}(C)+\max \{0, \varrho(\gamma)\}$ if $\bar{\gamma}(C) \times S$.

For $\varrho(\gamma) \leqq 0$ Equations (3.6) and (3.7) may be strengthened to read $\underline{\operatorname{deg}}_{u p^{\gamma}\left(s^{\gamma}-1\right) \mid v} \tau_{\gamma} Y_{\gamma}(C) \geqq \underline{\operatorname{deg}}_{u \mid p^{\gamma}\left(s^{\gamma}-1\right) v} Y_{\gamma}(C)$,

$\operatorname{deg}_{u \mid p^{\gamma}\left(s^{\gamma}-1\right) v} \tau_{\gamma} Y_{\gamma}(C) \geqq \underline{\operatorname{deg}}_{u \mid p^{\gamma}\left(s^{\gamma}-1\right) v} Y_{\gamma}(C)$.

Proof. Formulae (3.6-10) follow directly from the definition

$$
\tau_{\gamma}=\tau_{\gamma 1}+\tau_{\gamma 2}-\tau_{\gamma 1} \tau_{\gamma 2}
$$

where

$$
\tau_{\gamma 1}=t_{p^{\gamma}\left(s^{\gamma}-1\right)}^{\varrho(\gamma)-1}, \quad \tau_{\gamma^{2}}=t_{p^{\gamma} s^{\gamma}}^{\delta(\gamma)},
$$

and the following inequalities: if $\tau_{\gamma 1} Y_{\gamma}(C) \neq 0$,

$$
\begin{aligned}
& {\underline{\operatorname{deg}} u p^{\gamma}\left(s^{\gamma}-1\right) \mid v} \tau_{\gamma 1} Y_{\gamma}(C) \geqq \\
& \underline{\operatorname{deg}}_{u \mid p^{\gamma}\left(s^{\gamma}-1\right) v} \tau_{\gamma 1} Y_{\gamma}(C) \geqq\left\{\begin{array}{l}
\frac{\operatorname{deg}_{u p^{\gamma}\left(s^{\gamma}-1\right) \mid v} Y_{\gamma}(C),}{\text { if } \bar{\gamma}(C) \| S} \\
\operatorname{deg}_{u \mid p^{\gamma}\left(s^{\gamma}-1\right) v} Y_{\gamma}(C) \\
\text { if } \bar{\gamma}(C) X S,
\end{array}\right. \\
& \frac{\operatorname{deg}_{u p^{\gamma}\left(s^{\gamma}-1\right) \mid v}\left(1-\tau_{\gamma 1}\right) Y_{\gamma}(C) \geqq \underline{\operatorname{deg}}_{u \mid v p^{\gamma}\left(s^{\gamma}-1\right)} Y_{\gamma}(C)+\varrho(\gamma)+1}{\text { if } \bar{\gamma}(C) \nmid S ;}
\end{aligned}
$$

and if $\tau_{\gamma 2} Y_{\gamma}(C) \neq 0$,

$$
\begin{aligned}
& \operatorname{deg}_{u \mid p^{\gamma}\left(s^{\gamma}-1\right) v} \tau_{\gamma 2} Y_{\gamma}(C) \geqq \underline{\operatorname{deg}}_{u \mid v p^{\gamma}\left(s^{\gamma}-1\right)} Y_{\gamma}(C), \\
& \underline{\operatorname{deg}}_{u p^{\gamma}\left(s^{\gamma}-1\right) \mid v} \tau_{\gamma 2} Y_{\gamma}(C) \geqq \underline{\operatorname{deg}}_{u \mid v p^{\gamma}\left(s^{\gamma}-1\right)} Y_{\gamma}(C), \\
& \underline{\operatorname{deg}}_{u p^{\gamma}\left(s^{\gamma}-1\right) \mid v}\left(1-\tau_{\gamma 1}\right) \tau_{\gamma 2} Y_{\gamma}(C) \\
& \geqq \underline{\operatorname{deg}}_{u \mid v p^{\gamma}\left(s^{\gamma}-1\right)} Y_{\gamma}(C)+\max \{0, \varrho(\gamma)\} .
\end{aligned}
$$

To prove (3.11-13), we write

$$
Y_{\gamma}(C)=D^{-1}\left(p^{\gamma}, s^{\gamma}-1, u, v\right) \sum_{k=1}^{n} f_{k}\left(p^{\gamma}, s^{\gamma}-1\right) g_{k}(u, v)
$$


where

$f_{k}, \quad k=1,2, \ldots n, \quad$ are independent monomials, of degree $v(k)$, in $p^{\gamma}$ and $s^{\gamma}-1$,

$g_{k}, k=1,2, \ldots n$, are polynomials in $u$ and $v$,

$D \quad$ is a product of factors of the type ,

$$
\begin{aligned}
F_{L i}= & l_{L}^{\gamma 2}-\left(s^{\gamma}-1\right)^{2} M_{L i}^{2}-s^{\gamma 2} \mu_{L i}^{2}-m_{L i}^{2} \\
& +i \varepsilon\left(l_{L}^{\gamma 2}+\left(s^{\gamma}-1\right)^{2} M_{L i}^{2}+s^{\gamma 2} \mu_{L i}^{2}+m_{L i}^{2}\right)
\end{aligned}
$$

with

$$
l_{L}^{\gamma}=P_{L}\left(p^{\gamma}\right)+U_{L}(u)+V_{L}(v) .
$$

We note that $P_{L}\left(p^{\gamma}\right), \mu_{L i}^{2}$ and $M_{L i}^{2}$ can only be non-zero for $L \in \bar{\gamma}(C)$ or $L \in \bar{\lambda}(C)$, $\lambda$ a maximal element of $C$ contained in $\gamma$, with $\bar{\lambda}(C) \nmid S, \bar{\gamma}(C) \| S$. Moreover $V_{L}(v)=0$ for $L \in \bar{\gamma}(C) \| S$ and $V_{L}(v) \neq 0$ for $L \in \bar{\gamma}(C) \nmid S$. It is then straightforward to verify the following relations (abbreviations: $Y_{\gamma}=Y_{\gamma}(C), \bar{\gamma}=\bar{\gamma}(C), \operatorname{deg}_{u}=\operatorname{deg}_{u \mid v p^{\gamma}\left(s^{\gamma}-1\right)}$, $\left.\underline{\operatorname{deg}}_{u p^{\gamma}\left(s^{\gamma}-1\right)}=\underline{\operatorname{deg}}_{u p^{\gamma}\left(s^{\gamma}-1\right) \mid v}\right)$ :

$$
\begin{aligned}
& \underline{\operatorname{deg}}_{u p^{\gamma}\left(s^{\gamma}-1\right)} Y_{\gamma}=\underline{\operatorname{deg}}_{u p^{\gamma}\left(s^{\gamma}-1\right)} D^{-1}+\min _{k}\left\{v(k)+\underline{\operatorname{deg}}_{u} g_{k}\right\}, \\
& \underline{\operatorname{deg}}_{u} Y_{\gamma}=\underline{\operatorname{deg}}_{u} D^{-1}+\min _{k}\left\{v(k)+\underline{\operatorname{deg}}_{u} g_{k}\right\}, \\
& \underline{\operatorname{deg}}_{u p^{\gamma}\left(s^{\gamma}-1\right)} \tau_{\gamma 1} Y_{\gamma} \\
& \geqq \min _{\nu(k) \leqq \varrho(\gamma)-1}\left\{\underline{\operatorname{deg}}_{u p^{\gamma}\left(s^{\gamma}-1\right)} t_{p^{\gamma}\left(s^{\gamma}-1\right)}^{(\gamma)-1-v(k)} D^{-1}+v(k)+\underline{\operatorname{deg}}_{u} g_{k}\right\} \\
& (\equiv 0 \quad \text { if } \quad v(k)>\varrho(\gamma)-1 \text { for all } k) \text {, } \\
& \underline{\operatorname{deg}}_{u} \tau_{\gamma 1} Y_{\gamma} \geqq \min _{v(k) \leqq \varrho(\gamma)-1}\left\{\underline{\operatorname{deg}}_{u} t_{p^{\gamma}\left(s^{\gamma}-1\right)}^{(\gamma)-1(k)} D^{-1}+\underline{\operatorname{deg}}_{u} g_{k}\right\} \\
& (\equiv 0 \quad \text { if } \quad v(k)>\varrho(\gamma)-1 \text { for all } k), \\
& \underline{\operatorname{deg}}_{u p^{\gamma}\left(s^{\gamma}-1\right)}\left(1-\tau_{\gamma 1}\right) Y_{\gamma} \\
& \geqq \min _{k}\left\{\underline{\operatorname{deg}}_{u p^{\gamma}\left(s^{\gamma}-1\right)}\left(1-t_{p^{\gamma}\left(s^{\gamma}-1\right)}^{(\gamma)-1(k)}\right) D^{-1}+v(k)+\underline{\operatorname{deg}}_{u} g_{k}\right\}, \\
& \underline{\operatorname{deg}}_{u p^{\gamma}\left(s^{\gamma}-1\right)} t_{p^{\gamma}\left(s^{\gamma}-1\right)}^{\varrho(\gamma)-1(k)} D^{-1} \geqq \underline{\operatorname{deg}}_{u p^{\gamma}\left(s^{\gamma}-1\right)} D^{-1}, \\
& \operatorname{deg}_{u} t_{p^{\gamma}\left(s^{\gamma}-1\right)}^{(\gamma)-1-v(k)} D^{-1} \\
& \geqq \begin{cases}\operatorname{deg}_{u p^{\gamma}\left(s^{\gamma}-1\right)} D^{-1}-\varrho(\gamma)+1+v(k) & \text { if } \bar{\gamma} \| S \\
\underline{\operatorname{deg}}_{u} D^{-1} & \text { if } \bar{\gamma} \Varangle S,\end{cases} \\
& \underline{\operatorname{deg}}_{u p^{\gamma}\left(s^{\gamma}-1\right)}\left(1-t_{p^{\gamma}\left(s^{\gamma}-1\right)}^{\varrho(\gamma)-v(k)}\right) D^{-1} \\
& \geqq \varrho(\gamma)-v(k)+\operatorname{deg}_{u} D^{-1} \text { if } \bar{\gamma} \nmid S \text {. }
\end{aligned}
$$

Since, by hypothesis, $\tau_{\gamma 1} Y_{\gamma} \neq 0$, we have $v(k) \leqq \varrho(\gamma)-1$ for some $k$, and $(3.11-12)$ follow from (3.24-25), combined with (3.21-22). Inequality (3.13) follows from (3.26) and (3.23), comparing with (3.20). 
To obtain (3.14), from which (3.15) follows immediately, we simply observe that differentiating with respect to $p^{\gamma}$ and $s^{\gamma}$ and setting $p^{\gamma}=0=s^{\gamma}$ cannot lower $\operatorname{deg}_{u}$ of $D^{-1} f_{k} g_{k}$, since the relevant denominator factors have non-zero mass terms for $s^{\gamma}=0$. This fact also permits us to use essentially the same argument which led to (3.13) to obtain (3.16).

With the aid of Lemma 3.2, one obtains the following infrared power-counting inequalities for $Y_{\gamma}(C)$ :

Lemma 3.3. For $\Gamma, S, C$, and $1 P I \gamma \in C$ as in Lemma 3.2,

$$
\begin{aligned}
& \operatorname{deg}_{u p^{\gamma}\left(s^{\gamma}-1\right) \mid v} Y_{\gamma}(C) \geqq \varrho(\gamma)-M_{S}(\gamma) \text { for } \bar{\gamma}(C) \| S, \\
& \underline{\operatorname{deg}}_{u \mid p^{\gamma}\left(s^{\gamma}-1\right) v} Y_{\gamma}(C) \geqq-M_{S}(\gamma)+1 \text { for all } \gamma \in C,
\end{aligned}
$$

where

$$
M_{S}(\gamma)=4 \sum_{\substack{\lambda \in C \\ \lambda \subseteq \gamma \\ \bar{\lambda}(C) \| S}} \text { (no. of independent loops of } \bar{\lambda}(C) \text { ) }
$$

and $\stackrel{*}{\text { means }} \geqq$ with the r.h.s. $=0$ if $M_{S}(\gamma)=0$.

Lemma 3.4. Let $\Gamma, S$, and $C$ be as in Lemma 3.2. Let $\lambda$ be a maximal element of $C$ properly contained in $\gamma \in C \cup\{\hat{\Gamma}\}$. Then

$$
\begin{aligned}
& \underline{\operatorname{deg}}_{u \mid v p^{\gamma}\left(s^{\gamma}-1\right)} S_{\gamma} \tau_{\lambda} Y_{\gamma}(C) \geqq-M_{S}(\lambda)+1, \\
& \underline{\operatorname{deg}}_{u \mid v p^{\gamma}\left(s^{\gamma}-1\right)} S_{\gamma}\left(1-\tau_{\lambda}\right) Y_{\lambda}(C) \geqq-M_{S}(\lambda)+1, \\
& \underline{\operatorname{deg}}_{u p^{\gamma}\left(s^{\gamma}-1\right) \mid v} S_{\gamma} \tau_{\lambda} Y_{\lambda}(C) \geqq \max \{0, \varrho(\lambda)\}-M_{S}(\lambda) \text { for } \bar{\lambda}(C) \| S, \\
& \underline{\operatorname{deg}}_{u p^{\gamma}\left(s^{\gamma}-1\right) \mid v} S_{\gamma}\left(1-\tau_{\lambda}\right) Y_{\lambda}(C) \geqq \max \{0, \varrho(\lambda)\}-M_{S}(\lambda) \\
& \quad \text { for } \bar{\lambda}(C) \times S, \quad \bar{\gamma}(C) \| S .
\end{aligned}
$$

Proof of Lemmas 3.3 and 3.4. We adopt here the same notational conventions as in the proof of Lemma 3.2 .

The proof is by mathematical induction, with the inductive hypothesis that Lemma 3.3 holds for all maximal elements of $C$ contained in a given $\gamma \in C$. We shall show that (3.27-3.32) are valid for $\gamma$. Observe that for minimal $\gamma \in C$,

$$
Y_{\gamma}=I_{\bar{\gamma}(C)}
$$

and, for all $\gamma \in C$,

$$
\begin{aligned}
& \operatorname{deg}_{u p^{\gamma}\left(s^{\gamma}-1\right.}{ }^{\prime} I_{\gamma} \geqq r(\gamma)-M_{S}(\bar{\gamma}) \text { if } \bar{\gamma} \| S, \\
& \operatorname{deg}_{u} I_{\bar{\gamma}} \geqq 0 \stackrel{*}{\geqq}-M_{S}(\bar{\gamma})+1 .
\end{aligned}
$$

For minimal $\gamma,(3.34-35)$ imply (3.27-28). 
We now proceed to the verification of $(3.27-32)$, supposing that $\lambda$ is a maximal element of $C$ contained in $\gamma \in C[\gamma \in C \cup\{\hat{\Gamma}\}$ in the case of Lemma 3.4]. By the inductive hypothesis and Lemma 3.2,

$$
\begin{aligned}
& \quad \underline{\operatorname{deg}}_{u} \tau_{\lambda} Y_{\lambda} \geqq-M_{S}(\lambda)+1, \\
& \frac{\operatorname{deg}_{u}}{\operatorname{deg}_{u p^{\gamma}\left(s^{\gamma}-1\right)}}\left(1-\tau_{\lambda}\right) Y_{\lambda} \geqq-M_{S}(\lambda)+1, \\
& \underline{\operatorname{deg}}_{u p^{\gamma}\left(s^{\gamma}-1\right)}\left(1-\tau_{\lambda}\right) Y_{\lambda} \geqq \max \{0, \varrho(\lambda)\}-M_{S}(\lambda) \quad \text { if } \quad \bar{\lambda} \| S,
\end{aligned}
$$

We note that $\tau_{\lambda} Y_{\lambda}$ and $\left(1-\tau_{\lambda}\right) Y_{\lambda}$ may both be written in the form (3.17). Clearly the only way that $\operatorname{deg}_{u}$ of these expressions could be lowered by the $S_{\gamma}$ substitutions, $p^{\lambda} \rightarrow p^{\lambda}\left(p^{\gamma}, \overline{u, v}\right), s^{\lambda} \rightarrow s^{\gamma}$, is for some denominator factor (3.18) to have $M_{L i}^{2}=0=m_{L i}^{2}=\mu_{L i}^{2}$ and $k_{L}^{\lambda} \notin S, k_{L}^{\gamma} \in S$. But this is not possible and hence (3.29-30) follow from (3.36-37). Similarly, $\operatorname{deg}_{u p^{\lambda}\left(s^{\lambda}-1\right)} Y_{\lambda}$ (but not $\operatorname{deg}_{u p^{\lambda}\left(s^{\lambda}-1\right)} \tau_{\lambda} Y_{\lambda}$ ) could be lowered if $m_{L i}^{2}=0=\mu_{L i}^{2}, k_{L}^{\lambda} \notin S, k_{L}^{\gamma} \in \bar{S}$, but only if the internal parts of $S_{\gamma} p_{j}^{\lambda}$ are not in $S$ (for $\gamma=\hat{\Gamma}$, this means $S_{\Gamma} p^{\lambda} \notin S$ ). Hence (3.31-32) follow from (3.38-39).

To complete the inductive proof, we combine (3.34-35) with (3.29-32), using the relations

$$
\begin{gathered}
M_{S}(\gamma)=M_{S}(\bar{\gamma})+\sum_{\substack{\max \lambda \in C \\
\lambda \subset \gamma}} M_{S}(\lambda), \\
\varrho(\gamma) \leqq r(\bar{\gamma})+\sum_{\substack{\max \lambda \in C \\
\lambda \subset \gamma}} \varrho(\lambda)
\end{gathered}
$$

to obtain $(3.27-28)$.

Proof of Theorem 3.1. Let $\Gamma$ be a connected diagram. From Theorem 2, it is sufficient to show that for arbitrary basis $u_{1} \ldots u_{a} v_{1} \ldots v_{b}$ of $\mathscr{L}(\Gamma)$, the inequalities (2.13-14) are satisfied for $R_{\hat{\Gamma} \varepsilon}$. The first of these follows from the fact that $\tau_{\gamma}$ fulfills all of the criteria for a legitimate subtraction operator in the sense of Ref. [13], with the exception of property (iv), which is not relevant to the verification of (2.13). We now verify (2.14), using Lemmas 3.1 to 3.4 .

Let $C$ be a forest of $\hat{\Gamma}$ which is complete with respect to $S$, the subspace of spanned by $u_{1} \ldots u_{a}$. We wish to show

$$
\underline{\operatorname{deg}}_{u \mid v} R_{\hat{\Gamma} \varepsilon}(C)+4 a>0 \text {. }
$$

First of all, we have

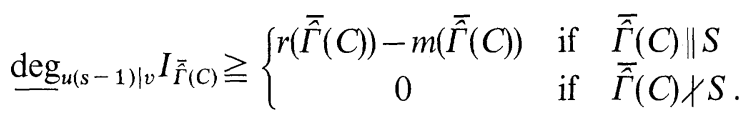

Moreover, if $V(\gamma)$ is a reduced vertex of $\bar{\Gamma}(C)$, we have as a consequence of (3.29-32),

$$
\begin{aligned}
\underline{\operatorname{deg}}_{u(s-1) \mid v} S_{\Gamma} f_{\gamma} Y_{\gamma}(C) & \\
\geqq \max \{0, \varrho(\gamma)\} & -M_{S}(\gamma) \quad \text { if } \quad \overline{\hat{\Gamma}}(C) \| S, \\
\underline{\operatorname{deg}}_{u(s-1) \mid v} S_{\Gamma} f_{\gamma} Y_{\gamma}(C) & \geqq \operatorname{deg}_{u \mid v(s-1)} S_{\Gamma} \tau_{\gamma} Y_{\gamma}(C) \\
& \geqq-M_{S}(\gamma)+1 \quad \text { if } \quad \bar{\Gamma}(C) \times S .
\end{aligned}
$$


Combining (3.43-45) and using

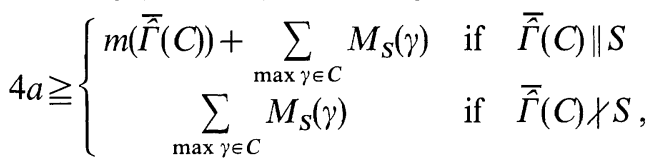

with equality only if the right-hand side does not vanish, we obtain

$$
\begin{gathered}
\underline{\operatorname{deg}}_{u \mid v} R_{\hat{\Gamma} \varepsilon}(C)+4 a \geqq \operatorname{deg}_{u(s-1) \mid v} R_{\hat{\Gamma} \varepsilon}(C)+4 a \\
\begin{cases}\geqq r(\overline{\hat{\Gamma}}(C)) & \text { if } \overline{\bar{\Gamma}}(C) \| S \\
>0 & \text { if } \quad \overline{\hat{\Gamma}}(C) \nmid S .\end{cases}
\end{gathered}
$$

Since by hypothesis $r(\bar{\Gamma}(C))$ is positive, inequality (3.42), and hence (2.14) are established.

We now turn to our second main convergence theorem.

Theorem 3.2. Let $\Gamma$ be a 1 PI diagram, with augmented diagram $\hat{\Gamma}$ and subtracted integrand $R_{\Gamma \varepsilon}$ given (2.7). Suppose that for every set $\{\gamma\}$ of mutually disjoint, nontrivial $1 P I$ subgraphs of $\hat{\Gamma}$ such that $\hat{\Gamma} /\{\gamma\}$ contains no q-lines, the inequality

$$
r(\hat{\Gamma} /\{\gamma\})+\sum_{\gamma} \max \{0, \varrho(\gamma)\}>0
$$

is satisfied. Then the integral

$$
\int d k R_{\Gamma \varepsilon}(p, k, s), \quad 0 \leqq s \leqq 1
$$

converges absolutely for arbitrary $\varepsilon>0$ and nonexceptional $p$ (Euclidean sense).

Proof. We must verify the convergence conditions of Theorem 2 . The ultraviolet criterion, (2.13), follows immediately from the theorem of Ref. [13]. To check (2.14), suppose that $u_{1} \ldots u_{a}, v_{1} \ldots v_{m-a}$ are linearly independent elements of $\mathscr{L}(\Gamma)$ with $\partial(u, v) / \partial(k) \neq 0$. By Lemma 3.1, it will be sufficient to show

$$
\underline{\operatorname{deg}}_{u \mid v} R_{\Gamma \varepsilon}(C)+4 a>0
$$

for arbitrary forest $C$ which is complete with respect to $S$, the space of linear combinations of $u_{1} \ldots u_{a}, p_{1} \ldots p_{u}$.

We first observe that Lemmas 3.2-3.4 remain valid for fixed external momenta. The only modification in the proofs is the addition of a term linear homogeneous in $p$ (distinguished from $p^{\gamma}$ !) in the expression for $l_{L}^{\gamma}$ in (3.18). Like the possibly non-zero mass $m_{L i}^{2}$, such a term does not affect the infrared power-counting estimates. In particular,

$$
\underline{\operatorname{deg}}_{u \mid v} \tau_{\Gamma} Y_{\Gamma}(C) \geqq \underline{\operatorname{deg}}_{u \mid p^{\Gamma}\left(s^{\Gamma}-1\right) v} \tau_{\Gamma} Y_{\Gamma}(C) \geqq-M_{S}(\Gamma)+1 .
$$

Since

$$
\begin{aligned}
4 a & \geqq M_{S}(\Gamma) \\
a & >0,
\end{aligned}
$$

relation (3.51) implies

$$
\underline{\operatorname{deg}}_{u \mid v} \tau_{\Gamma} Y_{\Gamma}(C)+4 a>0
$$

It remains to prove

$$
\underline{\operatorname{deg}}_{u \mid v} Y_{\Gamma}(C)+4 a>0 \text {. }
$$


It is easy to see that if $\bar{\Gamma}(C) \| S$, the lines (if any) whose momenta are linear combinations of $u_{1} \ldots u_{a}$ are those of a reduced diagram $\hat{\Gamma} /\{\gamma\}$ containing no $q$-lines and, moreover, $m(\hat{\Gamma} /\{\gamma\})$ is the number of such momenta which are linearly independent (here is where the non-exceptionality of the external momenta is important). Note that $\hat{\Gamma} /\{\gamma\}$ is in fact $\Gamma$ reduced by a connected subgraph which includes all external vertices. From here on the proof of (3.53) coincides with that of (3.42).

\section{Normal Products}

The convergence theorems of the preceding section can be applied to define Green functions of normal products $N_{\delta}^{\varrho}\left[Q^{(0)}(x)\right]$ and differential vertex operations (integrated normal products) $\Delta_{\delta}^{\varrho}\left[Q^{(0)}\right] \equiv \int d x N_{\delta}^{o}\left[Q^{(0)}(x)\right]$, where $Q^{(0)}$ is a formal product of free fields $\phi_{i}^{(0)}$, their derivatives and a non-negative integer power of $s$ or $(s-1)$. It is assumed that the fields $\phi_{i}^{(0)}$ have momentum-space two-point functions $\Delta_{i j}(p, s)$ of the type (2.1), with upper and lower degrees related to the naive UV and IR dimensions of the $\phi_{i}^{(0)}$ by

$$
\begin{aligned}
\overline{\operatorname{deg}}_{p s} \Delta_{i j} & =\bar{d}_{i}+\bar{d}_{j}-4 \\
\underline{\operatorname{deg}}_{p(s-1)} \Delta_{i j} & =\underline{d}_{i}+\underline{d}_{j}-4,
\end{aligned}
$$

where $\bar{d}_{i}=\overline{\operatorname{dim}} \phi_{i}^{(0)}, \underline{d}_{i}=\underline{\operatorname{dim}} \phi_{i}^{(0)}$, so that in particular

$$
\begin{aligned}
\bar{d}_{i} & =\frac{1}{2}\left(\overline{\operatorname{deg}}_{p s} \Delta_{i i}+4\right) \\
\underline{d}_{i} & =\frac{1}{2}\left(\underline{\operatorname{deg}}_{p(s-1)} \Delta_{i i}+4\right) .
\end{aligned}
$$

The positivity of $\underline{d}_{i}$ follows from the requirement that $\Delta_{i i}$ be well defined as a distribution. For reasons which will become clear shortly, we restrict ourselves to $\phi_{i}^{(0)}$ such that $\underline{d}_{i} \geqq \bar{d}_{i}$. In the definition of $N_{\delta}^{\varrho}\left[Q^{(0)}(x)\right], \delta$ and $\varrho$ are restricted by

$$
\delta=\overline{\operatorname{dim}} Q^{(0)}+\text { non-negative integer }
$$

$\varrho=\operatorname{dim} Q^{(0)}-$ non-negative integer

$0 \leqq \varrho \leqq \delta$.

We define, for $\varepsilon>0$,

$$
\left\langle 0\left|T \prod_{i=1}^{n+1} N_{\bar{\delta}_{2}}^{\varrho_{2}}\left[Q_{i}^{(0)}\left(x_{i}\right)\right] \prod_{j=1}^{r} \Delta_{\eta_{j}}^{\sigma_{j}}\left[M_{j}^{(0)}\right]\right| 0\right\rangle_{\varepsilon}^{\text {conn }}
$$

by the generalized Wick expansion (expansion in Feynman diagrams, with external vertices corresponding to $N_{\delta_{i}}^{e_{i}}\left[Q_{i}^{(0)}\left(x_{i}\right)\right]$ and internal vertices corresponding to $\Delta_{\eta_{j}}^{\sigma_{j}}\left[M_{j}\right]$, supplemented by the forest formula (2.7), with subtraction degrees

$$
\begin{aligned}
& \delta(\gamma)=4-\sum_{E} \bar{d}_{E}+\sum_{V_{i} \in \gamma}^{\text {ext }}\left(\delta_{i}-4\right)+\sum_{V_{\jmath} \in \gamma}^{\text {int }}\left(\eta_{j}-4\right) \\
& \varrho(\gamma)=4-\sum_{E} \underline{d}_{E}+\sum_{V_{\imath} \in \gamma}^{\text {ext }}\left(\varrho_{i}-4\right)+\sum_{V_{\jmath} \in \gamma}^{\text {int }}\left(\sigma_{j}-4\right)
\end{aligned}
$$


where

$$
\begin{aligned}
& \sum_{E}=\text { sum over external lines of } \gamma \\
& \sum_{V_{i} \in \gamma}^{\text {ext } / \text { int }}=\text { sum over external/internal vertices of } \gamma .
\end{aligned}
$$

Note that $\varrho(\gamma) \leqq \delta(\gamma)$.

The existence theorem for Green functions (4.4) is the following:

Theorem 4. If $\sigma_{j} \geqq 4, j=1,2, \ldots r$, then the connected Green function (4.4) exists as a tempered distribution in $x_{1} \ldots x_{n}$, and, for non-exceptional momenta (Euclidean sense), the vertex function

$$
\left\langle 0\left|T \prod_{i=1}^{n} N_{\delta_{i}}^{\varrho_{i}}\left[\tilde{Q}^{(0)}\left(p_{i}\right)\right] N_{\delta_{n+1}}^{\varrho_{n+1}}\left[Q_{n+1}^{(0)}(0)\right] \prod_{j=1}^{r} \Delta_{\eta_{j}}^{\sigma_{j}}\left[M_{j}^{(0)}\right]\right| 0\right\rangle_{\varepsilon}^{\text {prop }}
$$

(the tilde indicates Fourier transformation) exists as a complex-valued function.

Proof. From Theorems 3.1 and 3.2 it is sufficient to verify that for every set $\{\gamma\}$ of disjoint, non-trivial 1PI subdiagrams of a connected diagram, the inequality

$$
r(\hat{\Gamma} /\{\gamma\})+\sum_{\gamma} \max \{0, \varrho(\gamma)\}>0
$$

is satisfied.

From (2.3), we have, setting $\Lambda=\hat{\Gamma} /\{\gamma\}$,

$$
\begin{aligned}
r(\Lambda) & +\sum_{\gamma}\{0, \varrho(\gamma)\}=\sum_{L \in \Lambda}\left(\underline{D}_{L}+4\right)+\sum_{\substack{V \in A \\
V \in \Gamma}}\left(\underline{D}_{V}-4\right) \\
& +\sum_{\substack{\gamma \\
V_{0} \notin \gamma}}(\max \{0, \varrho(\gamma)\}-4)
\end{aligned}
$$

But

$$
\begin{aligned}
\sum_{L \in \Lambda}\left(\underline{D}_{L}+4\right) & =\sum_{k}\left(n_{k k}+\frac{1}{2} \sum_{l \neq k} n_{k l}\right)\left(\underline{D}_{k}+4\right)+4 n_{q} \\
& =\sum_{V \in \Lambda}^{\prime}\left(\sum_{k} v_{k}(V)+4 v_{q}(V)\right),
\end{aligned}
$$

where the last summation excludes $V=V_{0}$ or $V=V\left(\gamma_{0}\right)$ where $V_{0} \in \gamma_{0}$, and

$$
\begin{aligned}
n_{k l} & =\text { no. of } \Delta_{k l} \text { lines of } \Lambda \\
n_{q} & =\text { no. of } q \text {-lines of } \Lambda \\
v_{k}(V) & =\text { no. of } \Delta_{j k} \text { lines with } k \text { end at } V \\
\underline{D}_{k} & =\operatorname{deg}_{p(s-1)} \Delta_{k k} .
\end{aligned}
$$

Moreover, from (4.5) and

$$
\varrho_{V}-4 \geqq 0 \text { for all internal } V \in \Gamma,
$$

we obtain, for all reduced vertices $V(\gamma) \in \Lambda, V_{0} \notin \gamma$, with $v_{q}(V(\gamma))=0$,

$$
\varrho(\gamma)-4+\sum_{k} v_{k}(V(\gamma)) \underline{d}_{k} \geqq 0 .
$$


Substituting (4.9) and (4.11) into (4.8), we arrive at the relation

$$
\begin{aligned}
& r(\Lambda)+\sum_{\gamma} \max \{0, \varrho(\gamma)\} \\
& \geqq \sum_{\substack{V \in A \\
V \in \Gamma}}\left[\sum_{k \neq q} v_{k}(V) \underline{d}_{k}+\underline{D}_{V}+4\left(v_{q}(V)-1\right)\right] \\
& \quad+\sum_{\substack{\left.V(\gamma) \in \Lambda \\
v_{q}(V)\right)>0}}^{\prime}\left[\sum_{k \neq q} v_{k}(V(\gamma)) \underline{d}_{k}+\max \{0, \varrho(\gamma)\}+4\left(v_{q}(V(\gamma))-1\right)\right] \\
& \quad+\sum_{\substack{V=V_{0} \\
\text { or } V=V\left(\gamma_{0}\right) \\
V_{0} \in \gamma_{0}}}\left[\sum_{k \neq q} v_{k}(V) \underline{d}_{k}\right] .
\end{aligned}
$$

If $\Lambda$ contains $q$-lines, one of the first two summations in (4.12) must be positive [recall (4.10) and $\underline{d}_{k}>0$ ]. If $\Lambda$ has no $q$-lines, the last summation is positive.

This completes our proof of positivity of $\left.r(\Lambda)+\sum_{\gamma} \max \{0, \varrho, \gamma)\right\}$, and hence of the theorem. If, as is often the case, all elementary fields have $\underline{d}_{i} \geqq 1$, one can easily check that the hypothesis of the theorem may be relaxed slightly to include the possibility of one $\sigma_{j}$ equal to three.

We observe, finally, that Theorem 4 allows one to define Green functions in a wide class of interacting theories with both massive and massless particles:

$$
\begin{aligned}
& \left\langle T \prod_{i=1}^{n+1} N_{\delta_{i}}^{\varrho_{i}}\left[Q_{i}\left(x_{i}\right)\right] \prod_{j=1}^{r} \Delta_{\eta_{j}}^{\sigma_{j}}\left[M_{j}\right]\right\rangle_{\varepsilon}^{\mathrm{conn}} \quad\left(\sigma_{j} \geqq 4\right) \\
& \quad \equiv\left\langle 0\left|T \prod_{i=1}^{n+1} N_{\delta_{i}}^{\varrho_{i}}\left[Q_{i}^{(0)}\left(x_{i}\right)\right] \prod_{j=1}^{r} \Delta_{\eta_{j}}^{\sigma_{j}}\left[M_{j}^{(0)}\right] \exp i \Delta_{4}^{4}\left[\mathscr{L}_{\mathrm{eff} f}^{(0)}\right]\right| 0\right\rangle_{\varepsilon}^{\mathrm{conn}}
\end{aligned}
$$

where $Q_{i}$ and $M_{j}$ are formal products of the renormalized interacting fields (and factors of $s$ or $(s-1)$ ) with corresponding free-field products $Q_{i}^{(0)}$ and $M_{j}^{(0)}$, and $\mathscr{L}_{\text {eff } I}^{(0)}$ is a linear combination of free-field products (including $s$ or $(s-1)$ factors) with $\overline{\operatorname{dim}} \leqq 4, \underline{\operatorname{dim}} \geqq 4$. To every order in perturbation theory, the right-hand side of (4.12) is just a linear combination of terms of the form (4.4). Because of the assignment $\varrho_{V}=4$ for each interaction vertex, each of these terms is well defined as a tempered distribution.

Acknowledgements. Part of the foregoing research was done at the Instituto de Fisica of the Universidade de São Paulo and at the Max-Planck-Institut für Physik and Astrophysik in Munich. I would like to thank the Fundação de Amparo à Pesquisa do Estado de São Paulo and the MaxPlanck-Institut for making possible my visits to São Paulo and Munich. I am grateful to R. Köberle, M. Gomes, T. Clark, and P. Breitenlohner for a number of helpful discussions.

\section{References}

1. Zimmermann, W.: Commun. math. Phys. 15, 208 (1969)

2. Zimmermann, W.: Commun. math. Phys. 11, 1 (1969)

3. Lowenstein, J. H., Zimmermann, W.: Nucl. Phys. B86, 77 (1975)

4. Gomes, M., Schroer, B.: Phys. Rev. D10, 3525 (1974)

5. Lowenstein, J.H., Zimmermann, W.: Infrared Convergence of Feynman Integrals for the Massless $\mathrm{A}^{4}$-Model, Max-Planck-Institut preprint MPI-PAE/PTh 5/75 
6. Clark, T.: The Abelian Higgs Model in the Landau Gauge, Max-Planck-Institut preprint MPI$\mathrm{PAE} / \mathrm{PTh} 27 / 74$

7. Lowenstein, J.H., Zimmermann, W.: Commun. math. Phys. 44, 73 (1975)

8. Lowenstein, J.H., Speer, E.: New York University preprint NYP/TR 5/75

9. Clark, T., Lowenstein, J.H.: in preparation

10. Lowenstein, J.H.: New York University preprint NYU/TR 3/75

11. Becchi, C.: to be published

12. Clark, T., Rouet, A.: Proof of the Slavnov Identities in the Renormalized Georgi-Glashow Model, Max-Planck-Institut preprint MPI-PAE/PTh 17/75, in preparation

13. Gomes, M., Lowenstein, J.H., Zimmermann, W.: Commun. math. Phys. 39, 81 (1964)

Communicated by K. Symanzik

(Received August 5, 1975) 